\title{
Efficacy of High-Power Laser in Alleviating Pain and Improving Function of Patients With Patellofemoral Pain Syndrome: A Single-Blind Randomized Controlled Trial
}

\author{
Farshad Nouri' ${ }^{1}$, Seyed Ahmad Raeissadat ${ }^{2}$, Dariush Eliaspour ${ }^{1}$, Seyed Mansoor Rayegani ${ }^{1}$, Maryam Sadat \\ Rahimi $^{1 *}$, Behnaz Movahedi ${ }^{3}$
}

'Physical Medicine and Rehabilitation Research Center, Shahid Beheshti University of Medical Sciences, Tehran, Iran ${ }^{2}$ Clinical Research Development Center of Shahid Modarres Hospital \& Physical Medicine and Rehabilitation Research Center of Shahid Beheshti University of Medical Sciences, Tehran, Iran

${ }^{3}$ Department of Internal Medicine, Alborz Medical University, Karaj, Alborz, Iran

\author{
*Correspondence to \\ Maryam Sadat Rahimi, Physical \\ Medicine and Rehabilitation \\ Research Center, Shahid Beheshti \\ University of Medical Sciences, \\ Tehran, Iran \\ Tel: +982122724210; \\ Email: \\ maryam.sadat.rahimi@sbmu.ac.ir
}

Published online 15 December 2018

\begin{abstract}
Introduction: In this study, a single-blind and randomized controlled trial (RCT) for assessing the effectiveness of high-power (up to $12 \mathrm{~W}$ ) laser therapy (HPLT) on patients with patellofemoral pain syndrome (PFPS) was carried out.

Methods: Forty-four patients were randomly assigned to two treatment groups by generating random numbers with MATLAB 2014b software, where odd and even numbers were attributed to sham laser group (group A) and actual laser group (group B), respectively. Group B patients underwent HPLT with total dose of $300 \mathrm{~J} /$ session for 5 consecutive sessions separated by a 2-day interval. On the other hand, sham laser was applied to group A patients. Both groups had the same exercise therapy programs during the study period (3 months). The exercise therapy program included isometric knee exercise for 3 sets per day and 10 times in each set, with duration of 10 seconds per time and straight leg raise for 15 seconds 10 times a day. The group codes of patients were not revealed to subjects and data analyzer until completion of the study. Kujala, the Western Ontario and McMaster Universities Osteoarthritis Index (WOMAC) and visual analog scale (VAS) questionnaires were chosen as outcome measures. These questionnaires were completed at three points during the study; at the beginning of the study to obtain the pre-therapy conditions and one month and three months after the start of the study to evaluate post-therapy conditions.

Results: Two main analyses were conducted: within-group and between-group analyses. Withingroup analyses indicated significant improvements in respect to all measurements where pretherapy and post-therapy comparisons were conducted in both groups $(P<0.05)$. On the other hand, between-group comparisons did not reveal any statistically significant functional difference between group A and group B regarding the evaluative criteria $(P>0.05)$ except for pain VAS $(P$ $<0.05)$.

Conclusion: This study indicated that short-term HPLT accompanied by appropriate exercise regimen significantly decreased pain in patients with PFPS. But it was not recommended as an efficient modality in functional improvement. Also, it was observed that, in the short-term period of study, HPLT was a safe modality.

Keywords: High-power laser; Patellar chondromalacia; Patellofemoral pain syndrome; Exercise.
\end{abstract}

\section{Introduction}

Patellofemoral pain syndrome is one of the most common musculoskeletal disorders in athletes or active young adults. ${ }^{1-4}$ The pain existing in anterior knee or posterior patella without other knee pathologies is called PFPS. The prevalence of PFPS is about $1.5 \%$ to $7 \%$ in patients referring to medical centers with more occurrence in females and athletes. ${ }^{5,6}$ Although the main cause of this syndrome is unknown, some researchers believe that the pain can be attributed to some biomechanical, soft tissue or muscular abnormalities. ${ }^{7,8}$ This syndrome causes pain and functional impairment in the corresponding patients and may result in chronic pain and/or weaknesses in quadriceps muscles, if not treated well. One way to approach this syndrome is related to physical activities. In the literature, a number of randomized controlled

Please cite this article as follows: Nouri N, Raeissadat SA, Eliaspour D, Rayegani SM, Maryam Sadat Rahimi MS, Movahedi B. Efficacy of high-power laser in alleviating pain and improving function of patients with patellofemoral pain syndrome: A single-blind randomized controlled trial. J Lasers Med Sci. 2019;10(1):37-43. doi:10.15171/jlms.2019.06. 
trials (RCTs) and systematic reviews studied the efficacy of different treatments and interventions in PFPS that highlighted the importance of exercise therapy. ${ }^{8-10}$

Low-level laser therapy has been already applied to musculoskeletal pains such as carpal tunnel syndrome ${ }^{11,12}$ and knee osteoarthritis (OA). ${ }^{13}$ Recently, many studies have explored the efficacy of high power laser therapy in various musculoskeletal disorders such as low back pain, ${ }^{14}$ knee OA, ${ }^{15,16}$ lateral epicondylitis, ${ }^{17}$ subacromial impingement ${ }^{18}$ etc. Despite the growing use of highpower lasers in alleviating musculoskeletal pains and its confirmed physiologic effects, ${ }^{19,20}$ studies investigating the effect of high-power laser in pain management and function of patients with PFPS are very limited. Thus, the focus of this study is on analyzing the efficacy of high power laser in treatment of patients with PFPS. To do so, a single-blinded randomized clinical trial was designed in order to investigate the efficacy of high power laser in alleviating pain and improving functions of patients with PFPS.

\section{Methods}

Subjects

Patients referred to the outpatient physical medicine and rehabilitation (PM\&R) clinic of Shahid Modarres and Shohadaye Tajrish hospitals with anterior knee joint or posterior patella pain were evaluated using the most sensitive and specific tests (Shrug, Grind and Perkins test) to see if they were diagnosed with PFPS. ${ }^{21}$ Those with at least two positive tests were considered as potential subjects. It is worth noting that these tests were carried out by two PM\&R specialists. Of the total 78 patients diagnosed with PFPS, 34 were disqualified with regard to the inclusion and exclusion criteria. For the rest of patients, the research procedure, its main objectives and a description of high-power laser was elaborated and after obtaining written informed consents, they were included in the trial.

The inclusion criteria of this study were as follows. Patients aged 15 to 40 years referring to the outpatient PM\&R clinic of Shahid Modarres and Shohadaye Tajrish hospitals in year 2017 with positive diagnostic patellofemoral syndrome maneuvers including Shrug test, Grind test and Perkins test (at least 2 of them being positive) who had the symptoms for at least 3 months were included in this study. Having normal knee $x$-ray in 3 dimensions (A-P, lateral and patella view) was also an inclusion criterion.

On the other hand, patients aged younger than 15 years or older than 40 , history of knee surgery, a body mass index of greater than $30 \mathrm{~kg} / \mathrm{m}^{2}$, any systemic diseases such as diabetes mellitus, immunodeficiency or collagen vascular disorders were excluded. Furthermore, having a history of malignancy, recent knee trauma, any infection, any knee deformities or signs of knee OA in knee $\mathrm{x}$-ray, pregnancy or breastfeeding were considered as exclusion criteria.
Equipment

The active laser used in this study was a BTL-6000 high intensity laser with a wavelength of $1064 \mathrm{~nm}$ and a maximum power of $12 \mathrm{~W}$. For the active laser group, actual laser was utilized by a PM\&R specialist according to the protocol recommended by the device manufacturer. Based on the suggested protocol, Pain Relief program (with $10 \mathrm{~W}$ and $120 \mathrm{~J} / \mathrm{cm}^{2}$ ) for 120 seconds were exploited per therapy session. Pulsed laser in circulatory movements on patellar margins with duty factor of $25 \%$ was used while patients were supine with knee positioned in full extension (resting position of patellofemoral joint). The spot size was $0.8 \mathrm{~cm}^{2}$ and applicator distance from skin was $2 \mathrm{~cm}$. The same protocol by the same device with the same duration and positioning was also applied to the sham laser group where the device was in inactive mode and no laser beam was emitted. It should be pointed out that all 5 sessions were identical regarding the protocol, the positioning of laser and the therapy duration.

\section{Study Design, Randomization and Blinding}

Patients' personal and clinical information comprising age, gender, weight, body mass index (BMI), duration of symptoms, degree of joint stiffness and pain and their functional performance were gathered by the physician prior to the study using visual analog scale (VAS), Western Ontario and McMaster Universities Osteoarthritis Index (WOMAC) and Kujala questionnaires. VAS is a qualitative pain measurement tool that assigns integer numbers between zero and 10 to different pain levels, where zero means no pain and 10 means excruciating pain. ${ }^{22}$ Kujala is a questionnaire for quantifying the functionality of patients, where zero indicates complete disability and 100 denotes full functionality. Kujala questionnaire was translated into Persian and its validity and reliability for PFPS patients were confirmed. ${ }^{23}$ WOMAC is a questionnaire containing 24 questions for evaluating function, stiffness and pain in lower limb and knee. It was translated into Persian and its reliability and validity was shown in patients with knee OA..$^{24}$

The initial conditions of patients included in the study were examined by their baseline characteristics shown in Table 1. The data indicated that no significant difference was observed between the 2 groups regarding their pretherapy measurement values.

Afterwards, 44 patients were randomly assigned to 2 study groups through generating random numbers with MATLAB 2014b software by PM\&R specialist, that odd numbers were attributed to sham laser group (group A) and even numbers were assigned to actual laser group (group B). Neither the subjects nor the researchers know the group code of patients until the completion of data analyses. The sample size for our study was computed according to the work of van Linschoten et $\mathrm{a}^{25}$ that performed analogous comparisons.

Group B patients underwent high-power laser therapy 
Table 1. Baseline Characteristics of All Patients

\begin{tabular}{lccc}
\hline \multirow{2}{*}{ Variable } & \multicolumn{2}{c}{ Group } & \multirow{2}{*}{ P Value } \\
\cline { 2 - 3 } & Group A & Group B & \\
\hline Age $(y)$ & $31.43 \pm 6.72$ & $35.29 \pm 3.27$ & 0.07 \\
Gender & & & \\
Male & 6 & 6 & - \\
\hline Female & 14 & 14 & - \\
BMI & $23.26 \pm 2.84$ & $23.52 \pm 3.99$ & 0.84 \\
Pre-therapy comparisons & & & \\
VAS & $5.43 \pm 1.60$ & $6.50 \pm 1.34$ & 0.07 \\
Pain WOMAC & $8.00 \pm 3.64$ & $8.86 \pm 4.02$ & 0.56 \\
Function WOMAC & $20.57 \pm 11.34$ & $21.93 \pm 9.31$ & 0.73 \\
Stiffness WOMAC & $2.43 \pm 2.17$ & $2.57 \pm 1.45$ & 0.84 \\
\hline Kujala & $82.21 \pm 7.10$ & $75.43 \pm 10.23$ & 0.053 \\
WOMAC scores & $31.00 \pm 15.70$ & $33.36 \pm 11.78$ & 0.66 \\
\hline
\end{tabular}

(HPLT) for 5 consecutive sessions separated by a 2-day interval. Each patient was exposed to 120 seconds of 10 -W laser with $120 \mathrm{~J} / \mathrm{cm}^{2}$ per therapy session. On the other hand, sham laser was utilized for group A patients who were not aware of the inactiveness of the laser device. In addition to the laser therapy program, both groups had the same exercise therapy programs during the study period (3 months). In both groups, we used the same exercise program. Our quadriceps muscle strengthening program included isometric knee exercise for 3 sets per day and 10 times in each set, with duration of 10 seconds per time and straight leg raising for 15 seconds 10 times a day, this exercise regime was to be performed during the 3-month study period. Patients were regularly contacted by the PM\&R specialist in order to ensure their compliance with the exercise therapy program and check for potential complications.

Two major follow-up milestones were defined at which patients were brought back to their corresponding clinics to reassess their pain and functional performances by the same VAS, Kujala and WOMAC questionnaires and the same physician; 1 month and 3 months after the start of the therapy program. The obtained data at each milestone were recorded in a spreadsheet software program for later statistical analyses.

\section{Statistics}

As mentioned previously, 3 different categories of data for each group were obtained during the study; Preintervention data, first month data and third month data. Thus, 2 types of statistical analyses become necessary; within-group and between-group analyses. Withingroup analyses check the efficacy of each laser therapy program by comparing the pre-therapy and post-therapy data within each group. To perform within-group analyses, paired sample t-tests were implemented using the Statistical Package for the Social Sciences (SPSS) version 22.

Between-group analyses, however, compare the efficacy of group A with group B with regard to their related improvements. To do so, three improvement values were calculated for each group by comparing pre-therapy and post-therapy conditions in each group. Subsequently, the obtained improvements values of group A were compared to their counterpart values of group B by independentsamples $t$ tests using the same SPSS software. In fact, the outcomes of between-group analyses determine whether a significant difference in the efficacy of the different therapy regimes exists.

The results were expressed as mean \pm standard deviation (SD) and a 0.05 significance level was considered.

Noteworthy, the input data of patients were gathered using Kujala, ${ }^{26}$ WOMAC $^{27}$ and VAS questionnaires. Since the acquired data were of ordinal type, the choice between parametric and nonparametric statistical tests was controversial ${ }^{28}$ and there is no consensus among the researchers ${ }^{29}$ in this regard. For example, for VAS data, Scott and Huskisson ${ }^{30}$ recommended using nonparametric methods, whereas Philip ${ }^{31}$ preferred parametric statistics. Comprehensive analyses conducted lately on Likert item data by Frost ${ }^{32}$ suggests that the choice is tie between the two alternatives regarding their protection against false positives and false negatives. Taking this inference into account and the fact that nonparametric tests have strict assumptions that cannot be disregarded, parametric tests were selected for this study.

\section{Results}

Participant flow diagram is illustrated in Figure 1.

As mentioned before, 44 patients were randomly allocated to two groups. Of these 44 patients, 4 refused to participate in the research; 2 because of not answering phone calls and 2 because of incompatibility of clinic schedules with their intervals. The mean BMI was almost the same between groups with $23.26 \pm 2.84$ for group A and $23.52 \pm 3.99$ for group $B(P>0.05)$.

The mean age of group A was $31.43 \pm 6.72$ and the mean age of group B was $35.29 \pm 3.27$.

Table 1 shows a summary of initial conditions for each group while the pre-therapy comparisons are also provided. Using independent sample $t$ tests, it can be seen that the starting conditions for all subjects between groups A and B are nearly the same.

As can be seen in Table 2, both sham and actual laser groups demonstrate significant improvements in all output measurements when pre-therapy values are compared to the post-therapy values $(P<0.05)$. However, there was no statistically significant difference between mean values of month 1 and month 3 in either treatment group implying that the attained improvements were maintained during the three-month period after active or sham laser therapy.

From VAS measures viewpoint, improvements in active laser group (group B) during trial period were statistically 


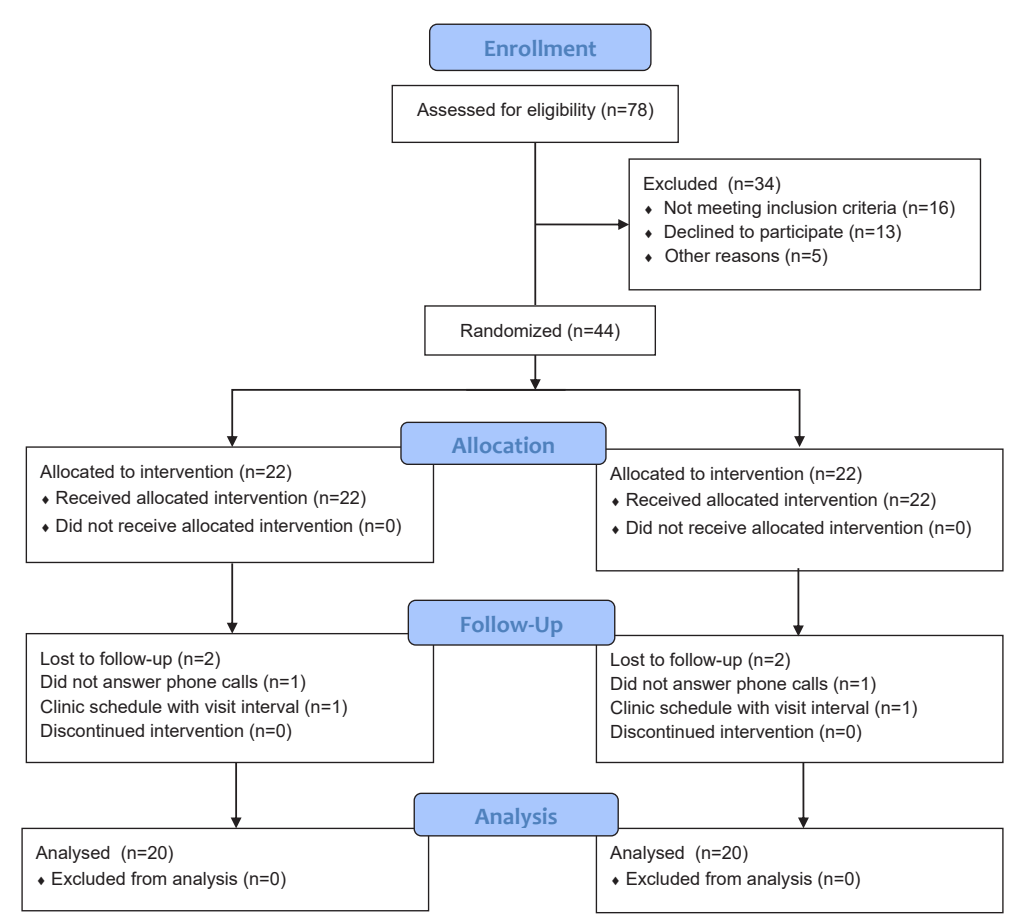

Figure 1. Participant Flow Diagram.

more significant than those of sham laser group $(P<0.05$; Figure 2).

Although improvements in the other measurements (Pain WOMAC, Function WOMAC, Stiffness WOMAC, Kujala and WOMAC scores) in active laser group were more than those of sham laser group, the differences were not proved to be statistically significant $(P>0.05)$ (Table 3).

Figure 3 provides visual comparisons of between-group and within-group improvements during the therapy program.

Post-therapy clinical outcomes for both actual and sham laser groups are shown in Table 4 . It is worth mentioning that no complications were reported during the study period in either group.

\section{Discussion}

The purpose of this study was to evaluate the efficacy of high-power laser in decreasing pain and improving functions of patients with PFPS. The study showed that the methods, the exercise alone (sham laser group) and exercise combined with high-power laser (active laser group) had effectiveness at follow-up moments. Actual laser outperformed sham laser with respect to pain relief while no significant difference in terms of functional

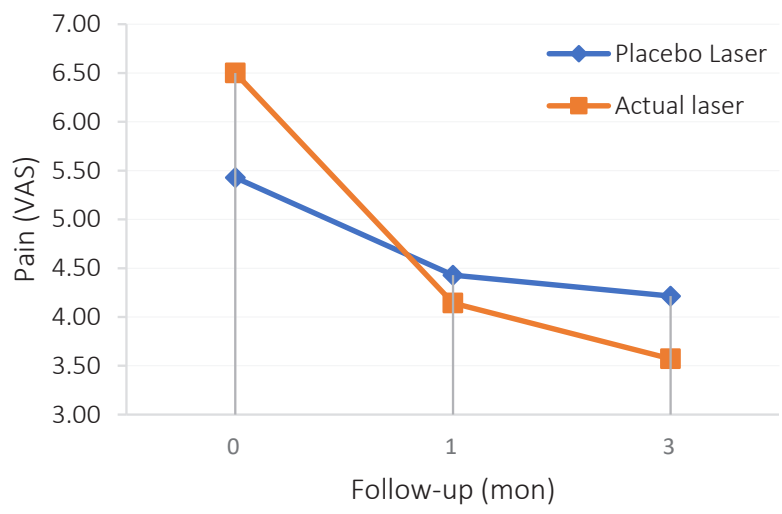

Figure 2. VAS Changes During the Therapy Program.

Table 2. Within-Group Comparisons Made by Paired-Samples $T$ Test ( $P$ Value)

\begin{tabular}{|c|c|c|c|c|c|c|}
\hline & \multicolumn{3}{|c|}{ Group A } & \multicolumn{3}{|c|}{ Group B } \\
\hline & 0 vs 1 & 0 vs 3 & 1 vs 3 & 0 vs 1 & 0 vs 3 & 1 vs 3 \\
\hline VAS & 0.002 & 0.011 & 0.385 & 0.000 & 0.001 & 0.293 \\
\hline Pain WOMAC & 0.005 & 0.005 & 0.567 & 0.001 & 0.004 & 0.373 \\
\hline Function WOMAC & 0.005 & 0.006 & 0.231 & 0.002 & 0.002 & 0.322 \\
\hline Stiffness WOMAC & 0.015 & 0.043 & 0.189 & 0.005 & 0.002 & 0.212 \\
\hline Kujala & 0.007 & 0.015 & 0.687 & 0.043 & 0.033 & 0.592 \\
\hline WOMAC scores & 0.003 & 0.006 & 0.901 & 0.001 & 0.001 & 0.427 \\
\hline
\end{tabular}


Table 3. Between-Group Comparisons of the Achieved Improvements Made by Independent-Samples $T$ Test (Between Group A and B) $(P$ Value)

\begin{tabular}{lccc}
\hline & $\begin{array}{c}\text { Improvements From Pre-therapy } \\
\text { Condition to Month } \mathbf{1}\end{array}$ & $\begin{array}{c}\text { Improvements From Pre-therapy } \\
\text { Condition to Month 3 }\end{array}$ & $\begin{array}{c}\text { Improvements From Month } \mathbf{1} \text { to Month } \mathbf{3} \\
\text { After the Therapy Initiation }\end{array}$ \\
\hline VAS & 0.03 & 0.03 & 0.54 \\
Pain WOMAC & 0.29 & 0.31 & 1 \\
Function WOMAC & 0.72 & 0.59 & 0.61 \\
Stiffness WOMAC & 0.90 & 0.26 & 0.09 \\
Kujala & 0.87 & 0.98 & 0.82 \\
WOMAC scores & 0.55 & 0.42 & 0.52 \\
\hline
\end{tabular}

improvement was observed.

Recently, many studies have evaluated the efficacy of HPLT in various musculoskeletal disorders such as low back pain, ${ }^{14}$ knee OA, ${ }^{15,16}$ lateral epicondylitis, ${ }^{17}$ subacromial impingement ${ }^{18}$ etc. Despite the mentioned studies about high-power laser in other musculoskeletal studies, the findings of this work regarding high-power laser did not reveal any significant superiority over sham laser, except for pain relief with VAS index that may be attributed to multifactorial nature of PFPS, difficult approachability to this joint or due to differences in protocols and/or doses. To the best knowledge of authors, it was the first study in the literature that considered high-power laser for PFPS. However, the use of low-power laser for this syndrome was observed in some studies. Rogvi-Hansen et al, ${ }^{33}$ for instance, used low-power laser combined with exercise regime for PFPS. They found the same findings in both control and intervention groups as ours except for pain relief measured by VAS index. The pain relief achieved by using laser is due to laser's biological effects on cellular
(A)

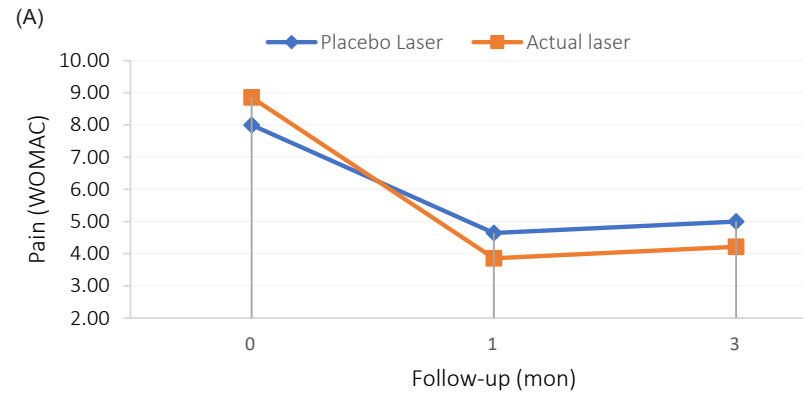

(C)

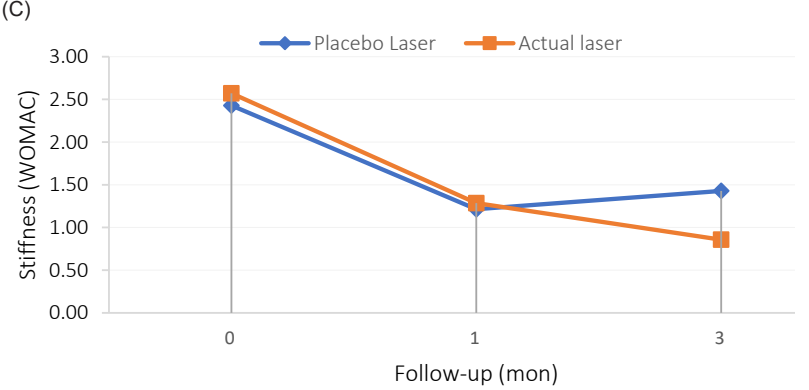

(E)

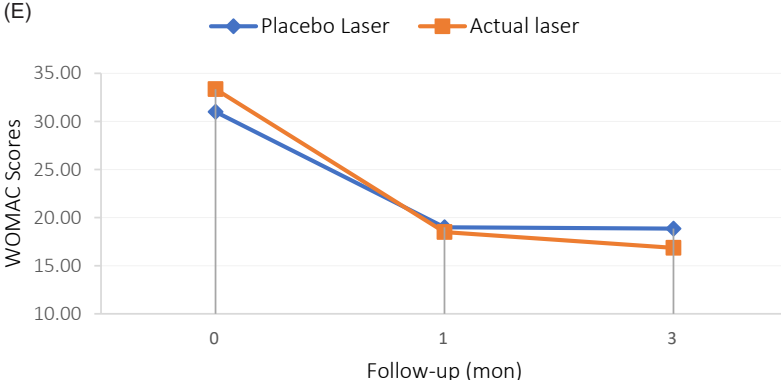

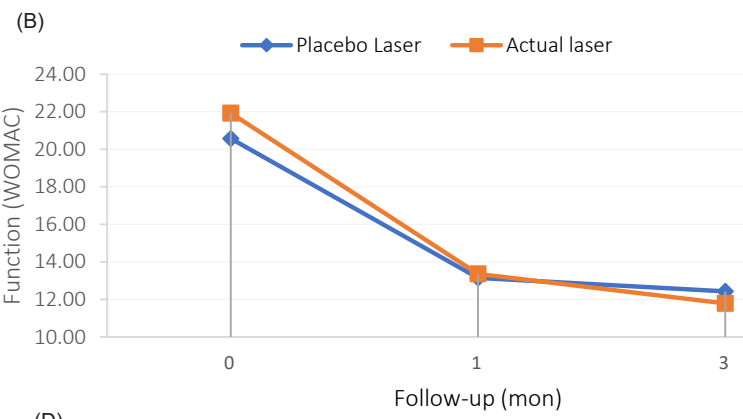

(D)

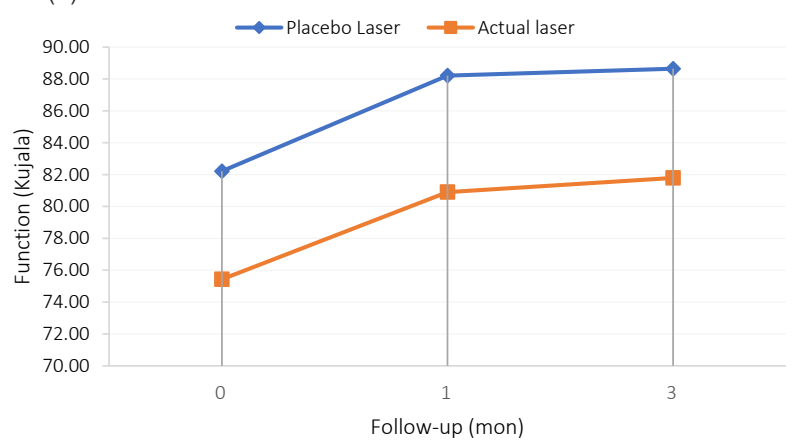

Figure 3. (A) Pain WOMAC, (B) Function WOMAC), (C) Stiffness WOMAC, (D) Function (Kujala), and (E) WOMAC Total Scores Changes for Each Group During the Therapy Program. 
Table 4. Post-therapy Clinical Outcomes of Both Groups

\begin{tabular}{|c|c|c|c|c|}
\hline \multirow{2}{*}{ Variables } & \multicolumn{2}{|c|}{ Group A (Sham Laser) } & \multicolumn{2}{|c|}{ Group B (Active Laser) } \\
\hline & 1st Month & 3rd Month & 1st Month & 3rd Month \\
\hline VAS & $4.43 \pm 1.28^{\mathrm{a}}$ & $4.21 \pm 1.58^{b}$ & $4.14 \pm 2.21^{\mathrm{c}, \mathrm{e}}$ & $3.57 \pm 2.68^{\mathrm{d}, \mathrm{f}}$ \\
\hline Pain WOMAC & $4.64 \pm 2.02^{\mathrm{a}}$ & $5.00 \pm 2.29^{b}$ & $3.86 \pm 3.16^{c}$ & $4.21 \pm 3.29^{\mathrm{d}}$ \\
\hline Function WOMAC & $13.14 \pm 6.43^{a}$ & $12.43 \pm 6.57^{b}$ & $13.36 \pm 9.73^{c}$ & $11.79 \pm 10.51^{\mathrm{d}}$ \\
\hline Stiffness WOMAC & $1.21 \pm 1.12^{\mathrm{a}}$ & $1.43 \pm 1.34^{\mathrm{b}}$ & $1.29 \pm 1.33^{c}$ & $0.86 \pm 1.51^{\mathrm{d}}$ \\
\hline Kujala & $88.21 \pm 5.99^{a}$ & $88.64 \pm 6.18^{\mathrm{b}}$ & $80.93 \pm 10.56^{c}$ & $81.79 \pm 9.82^{\mathrm{d}}$ \\
\hline WOMAC scores & $19.00 \pm 9.00^{\mathrm{a}}$ & $18.86 \pm 9.04^{b}$ & $18.50 \pm 13.11^{c}$ & $16.86 \pm 14.49^{d}$ \\
\hline
\end{tabular}

Data are shown as mean \pm standard deviation.

${ }^{\mathrm{a}} 1$ st month and ${ }^{\mathrm{b}} 3 \mathrm{rd}$ month values of group $\mathrm{A}$ that are significantly different from pre-therapy values of the same group by paired-samples $t$ test $(P<$ $0.05)$.

${ }^{c} 1$ st month and ${ }^{\mathrm{d}} 3 \mathrm{rd}$ month values of group B that are significantly different from pre-therapy values of the same group by paired-samples $t$ test $(P<$ $0.05)$.

e Improvements achieved in the first month of therapy of group B that are significantly different from the corresponding values of group A by independent-samples $t$ test $(P<0.05)$.

${ }^{\mathrm{f}}$ improvements achieved in the third month of therapy of Group B that are significantly different from the corresponding values of group A by independent-samples $t$ test $(P<0.05)$.

and tissue function, releasing endogen opium, thermal and anti-inflammatory effects which can propagate better in high-power lasers compared to low-power ones.

Since PFPS has a multifactorial nature, various factors including quadriceps weakness, tightness of hamstring, iliopsoas and gastrosoleus muscles, etc are investigated as its potential risk factors. ${ }^{34}$ Accordingly, a diversity of physical activities is proposed for treatment of PFPS. ${ }^{35-40}$ According to the obtained results, both groups showed significant improvements compared to the pre-therapy conditions. It was due to the identical exercise therapy that was employed by both groups. Thus, it can be concluded that it was an efficient regime for patients with PFPS, which is in accordance with the findings of similar investigations in the literature. Moreover, the efficacy of the exercise regime was improved when high-power laser was also included.

A limitation of this study can be attributed to the small number of subjects. Of the 44 potential subjects, 4 patients were excluded from the study because of their personal considerations, unrelated to the laser complication or so on. This limitation negatively affected the power of statistical tests conducted in the article. Another drawback can be attributed to the lack of articles in the literature considering the efficacy of HPLT in treating PFPS. Thus, we had difficulty in finding standard doses or durations for carrying out the HPLT. Further studies in the future are necessary to investigate the efficacy of HPLT with various doses and durations on an increased population of patients. Moreover, the physician was not blinded to the groups that might result in biased outcomes.

This study indicated that short-term HPLT accompanied by appropriate exercise regimen can significantly decrease pain in patients with PFPS, but it cannot assume as an efficient modality in functional improvement.

\section{Ethical Considerations}

This study was registred in Iranian Registry of Clinical Trials (IRCT) (Identifier: IRCT2017100713442N19; https://www.irct.ir). Informed consent was obtained from all individual participants included in the study.

\section{Conflict of Interests}

The authors declare no conflict of interest.

\section{References}

1. Jensen R, Kvale A, Baerheim A. Is pain in patellofemoral pain syndrome neuropathic? Clin J Pain. 2008;24(5):384394. doi:10.1097/AJP.0b013e3181658170

2. Evans RC. Illustrated Orthopedic Physical Assessment-EBook. Elsevier Health Sciences; 200m.

3. Nejati P, Forogh B, Moeineddin R, Baradaran HR, Nejati M. Patellofemoral pain syndrome in Iranian female athletes. Acta Med Iran. 2011;49(3):169-172.

4. Piazza L, Lisboa ACA, da Costa V, et al. Symptoms and functional limitations of patellofemoral pain syndrome patients. Rev Dor. 2012;13(1):50-54. doi:10.1590/S180600132012000100009

5. Glaviano NR, Kew M, Hart JM, Saliba S. Demographic and epidemiological trends in patellofemoral pain. Int J Sports Phys Ther. 2015;10(3):281-290.

6. McConnell J. The management of chondromalacia patellae: a long term solution. Aust J Physiother. 1986;32(4):215-223. doi:10.1016/s0004-9514(14)60654-1

7. Bentley G, Dowd G. Current concepts of etiology and treatment of chondromalacia patellae. Clin Orthop Relat Res. 1984(189):209-228.

8. Crossley K, Bennell K, Green S, McConnell J. A systematic review of physical interventions for patellofemoral pain syndrome. Clin J Sport Med. 2001;11(2):103-110.

9. Eng JJ, Pierrynowski MR. Evaluation of soft foot orthotics in the treatment of patellofemoral pain syndrome. Phys Ther. 1993;73(2):62-68; discussion 68-70.

10. Eburne J, Bannister G. The McConnell regimen versus 
isometric quadriceps exercises in the management of anterior knee pain. A randomised prospective controlled trial. Knee. 1996;3(3):151-153. doi:10.1016/09680160(96)00214-1

11. Raeissadat A, Reza Soltani Z. Study of Long Term Effects of Laser Therapy Versus Local Corticosteroid Injection in Patients with Carpal Tunnel Syndrome. J Lasers Med Sci. 201O;1(1):24-30. doi:10.22037/2010.v1i1.2134

12. Rayegani SM, Bahrami MH, Eliaspour D, et al. The effects of low intensity laser on clinical and electrophysiological parameters of carpal tunnel syndrome. J Lasers Med Sci. 2013;4(4):182-189.

13. Rayegani SM, Raeissadat SA, Heidari S, Moradi-Joo M. Safety and Effectiveness of Low-Level Laser Therapy in Patients With Knee Osteoarthritis: A Systematic Review and Meta-analysis. J Lasers Med Sci. 2017;8(Suppl 1):S12-s19. doi:10.15171/jlms.2017.s3

14. Alayat MS, Atya AM, Ali MM, Shosha TM. Long-term effect of high-intensity laser therapy in the treatment of patients with chronic low back pain: a randomized blinded placebo-controlled trial. Lasers Med Sci. 2014;29(3):10651073. doi:10.1007/s10103-013-1472-5

15. Stiglic-Rogoznica N, Stamenkovic D, Frlan-Vrgoc L, Avancini-Dobrovic V, Vrbanic TS. Analgesic effect of high intensity laser therapy in knee osteoarthritis. Coll Antropol. 2011;35 Suppl 2:183-185.

16. Kheshie AR, Alayat MS, Ali MM. High-intensity versus low-level laser therapy in the treatment of patients with knee osteoarthritis: a randomized controlled trial. Lasers Med Sci. 2014;29(4):1371-1376. doi:10.1007/s10103-0141529-0

17. Dundar U, Turkmen U, Toktas H, Ulasli AM, Solak O. Effectiveness of high-intensity laser therapy and splinting in lateral epicondylitis; a prospective, randomized, controlled study. Lasers Med Sci. 2015;30(3):1097-1107. doi:10.1007/ s10103-015-1716-7

18. Santamato A, Solfrizzi V, Panza F, et al. Short-term effects of high-intensity laser therapy versus ultrasound therapy in the treatment of people with subacromial impingement syndrome: a randomized clinical trial. Phys Ther. 2009;89(7):643-652. doi:10.2522/ptj.20080139

19. Bjordal JM, Couppe C, Chow RT, Tuner J, Ljunggren EA. A systematic review of low level laser therapy with locationspecific doses for pain from chronic joint disorders. Aust J Physiother. 2003;49(2):107-116.

20. Enwemeka CS, Parker JC, Dowdy DS, Harkness EE, Sanford LE, Woodruff LD. The efficacy of low-power lasers in tissue repair and pain control: a meta-analysis study. Photomed Laser Surg. 2004;22(4):323-329. doi:10.1089/ pho.2004.22.323

21. Nijs J, Van Geel C, Van der auwera C, Van de Velde B. Diagnostic value of five clinical tests in patellofemoral pain syndrome. Man Ther. 2006;11(1):69-77. doi:10.1016/j. math.2005.04.002

22. Wewers ME, Lowe NK. A critical review of visual analogue scales in the measurement of clinical phenomena. Res Nurs Health. 1990;13(4):227-236.

23. Negahban H, Pouretezad M, Yazdi MJ, et al. Persian translation and validation of the Kujala Patellofemoral Scale in patients with patellofemoral pain syndrome. Disabil Rehabil. 2012;34(26):2259-2263. doi:10.3109/0963 8288.2012.683480
24. Ebrahimzadeh $\mathrm{MH}$, Makhmalbaf $\mathrm{H}$, Birjandinejad A, Keshtan FG, Hoseini HA, Mazloumi SM. The Western Ontario and McMaster Universities Osteoarthritis Index (WOMAC) in Persian Speaking Patients with Knee Osteoarthritis. Arch Bone Jt Surg. 2014;2(1):57-62.

25. van Linschoten R, van Middelkoop M, Berger MY, et al. Supervised exercise therapy versus usual care for patellofemoral pain syndrome: an open label randomised controlled trial. BMJ. 2009;339:b4074. doi:10.1136/bmj. b4074

26. Kujala UM, Jaakkola LH, Koskinen SK, Taimela S, Hurme M, Nelimarkka O. Scoring of patellofemoral disorders. Arthroscopy. 1993;9(2):159-163.

27. McConnell S, Kolopack P, Davis AM. The Western Ontario and McMaster Universities Osteoarthritis Index (WOMAC): a review of its utility and measurement properties. Arthritis Rheum. 2001;45(5):453-461.

28. Mantha S, Thisted R, Foss J, Ellis JE, Roizen MF. A proposal to use confidence intervals for visual analog scale data for pain measurement to determine clinical significance. Anesth Analg. 1993;77(5):1041-1047.

29. Dexter F, Chestnut DH. Analysis of statistical tests to compare visual analog scale measurements among groups. Anesthesiology. 1995;82(4):896-902.

30. Scott J, Huskisson EC. Graphic representation of pain. Pain. 1976;2(2):175-184.

31. Philip BK. Parametric statistics for evaluation of the visual analog scale. Anesth Analg. 1990;71(6):710.

32. Frost J. Best Way to Analyze Likert Item Data: Two Sample T-Test versus Mann-Whitney. Minitab Blog; 2016. http:// blog.minitab.com/blog/adventures-in-statistics-2/bestway-to-analyze-likert-item-data:-two-sample-t-testversus-mann-whitney. Accessed November 20, 2017.

33. Rogvi-Hansen B, Ellitsgaard N, Funch M, Dall-Jensen M, Prieske J. Low level laser treatment of chondromalacia patellae. Int Orthop. 1991;15(4):359-361.

34. Halabchi F, Mazaheri R, Seif-Barghi T. Patellofemoral pain syndrome and modifiable intrinsic risk factors; how to assess and address? Asian J Sports Med. 2013;4(2):85-100.

35. Wise HH, Fiebert I, Kates JL. EMG Biofeedback as Treatment for Patellofemoral Pain Syndrome. J Orthop Sports Phys Ther. 1984;6(2):95-103.

36. Boucher JP, King MA, Lefebvre R, Pepin A. Quadriceps femoris muscle activity in patellofemoral pain syndrome. Am J Sports Med. 1992;20(5):527-532. doi:10.1177/036354659202000507

37. Steinkamp LA, Dillingham MF, Markel MD, Hill JA, Kaufman KR. Biomechanical considerations in patellofemoral joint rehabilitation. Am J Sports Med. 1993;21(3):438-444. doi:10.1177/036354659302100319

38. Rice MA, Bennett JG, Ruhling RO. Comparison of two exercises on VMO and VL EMG activity and force production. Isokinet Exerc Sci. 1995;5(2):61-67 doi:10.3233/ IES-1995-5201

39. Cerny K. Vastus medialis oblique/vastus lateralis muscle activity ratios for selected exercises in persons with and without patellofemoral pain syndrome. Phys Ther. 1995;75(8):672-683.

40. Powers CM. Patellar kinematics, part I: the influence of vastus muscle activity in subjects with and without patellofemoral pain. Phys Ther. 2000;80(10):956-964. 